\title{
Akademie Online
}

\section{Preis-Special für Späteinsteiger 2017}

Sie sind begeisterter Testhörer der Akademie-Online-Kurse und haben bisher den kompletten Jahreskurs nur noch nicht gebucht, weil Sie denken: im Sommer lohnt sich's nicht mehr? Dann kommt jetzt das richtige Einsteiger-Angebot für Sie: Seit dem 01.07.2017 buchen Sie das Akademie-Online-Jahresabo zum halben Preis!

Vom 01.07. bis 31.12.2017 warten insgesamt noch zwölf ärztliche Online-Kurse und sechs MTRA-Online-Kurse zu günstigen Konditionen auf Sie. Und das Beste daran ist: Als Halbjahresabonnent haben

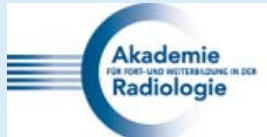

\section{AKADEMIE}

FÜR FORT- UND WEITERBILDUNG IN DER RADIOLOGIE

in der Deutschen Röntgengesellschaft e.V.
Sie ebenfalls Zugriff auf alle Kursaufzeichnungen ab dem 01.07.2017 und können verpasste Kurse noch einmal bequem für 14 bzw. 28 Tage nachträglich ansehen.

Für jedes besuchte Live-Webinar erhalten Sie eine Teilnahmebescheinigung. Alle Kurse sind mit 2 CME-Punkten der Akademie zertifiziert; alle ärztlichen Kurse werden i.d. R. zusätzlich mit jeweils 2 Fortbildungspunkten der Ärztekammer Hessen zertifiziert.
Eine Übersicht über die neuen Aktionspreise finden Sie in der unten stehenden Tabelle; die Tarife sind seit dem 16.06.2017 freigeschaltet. Alle Informationen, den aktuellen Kursplan und die Möglichkeit zur Anmeldung finden Sie unter www.drgakademie.de > Akademie Online.
Normales Jahresabo (Teilnahme und Zugang zum Streambereich für alle Ärzte- und MTRA-Kurse vom 01.01.2017-31.12.2017)
Aktionspreis Halbjahresabo (Teilnahme und Zugang zum Streambereich für alle Ärzte- und MTRA-Kurse vom 01.07.2017-31.12.2017)

\begin{tabular}{l|c|c|}
\hline $\begin{array}{l}\text { Fachärztin/Facharzt - Mitglied DRG/BDR/ÖRG } \\
\text { oder SRG-SSR }\end{array}$ & $200 \mathrm{EUR}$ & 100 EUR \\
\hline Fachärztin/Facharzt - ohne Mitgliedschaft & $800 \mathrm{EUR}$ & 200 EUR \\
\hline AiW - Mitglied DRG/BDR/ÖRG oder SRG-SSR & $160 \mathrm{EUR}$ & 40 EUR \\
\hline AiW - ohne Mitgliedschaft & $80 \mathrm{EUR}$ & 80 EUR \\
\hline Ärztin/Arzt in Erziehungsurlaub & $60 \mathrm{EUR}$ & 40 EUR \\
\hline MTRA - VMTB-Mitglied & $140 \mathrm{EUR}$ & 30 EUR \\
\hline MTRA - ohne Mitgliedschaft & & 70 EUR
\end{tabular}

